\title{
Understanding Nuclear Binding Energy with Strong and Electroweak Coupling Constants
}

\author{
U. V. Satya Seshavatharam ${ }^{1, *} \&$ S. Lakshminarayana ${ }^{2, *}$ \\ ${ }^{1}$ Honorary Faculty, I-SERVE, Survey no-42, Hitex road, Hitech city, Hyderabad-84, Telangana, India \\ ${ }^{2}$ Department of Nuclear Physics, Andhra University, Visakhapatnam-03, AP, India \\ * Corresponding Emails: vedakavi@serveveda.org (or) sln@auvsp.edu.in
}

\begin{abstract}
At nuclear scale, we present three heuristic relations pertaining to strong and electroweak coupling constants. With these relations, close to beta stability line, it is possible to study nuclear binding energy with a single energy coefficient of magnitude $\left(\frac{1}{\alpha_{s}}\right)\left[\frac{e^{2}}{4 \pi \varepsilon_{0} R_{0}}\right] \approx 10.0 \mathrm{MeV}$. With reference to up and down quark masses, it is also possible to interpret that, nuclear binding energy is proportional to the mean mass of $\left[\left(2 m_{u}+m_{d}\right)\right.$ and $\left.\left(m_{u}+2 m_{d}\right)\right] \approx 10.0 \mathrm{MeV}$.
\end{abstract}

Keywords: nuclear charge radius; strong coupling constant; Fermi's weak coupling constant; nuclear binding energy coefficient

\section{Introduction}

The modern theory of strong interaction is Quantum chromodynamics (QCD) [1]. It explores baryons and mesons in broad view with 6 quarks and 8 gluons. According to QCD, the four important properties of strong interaction are: 1) color charge;2) confinement; 3) asymptotic freedom [2];4) short-range nature $\left(<10^{-15} \mathrm{~m}\right)$. Color charge is assumed to be responsible for the strong force to act on quarks via the force carrying agent, gluon. Experimentally it is well established that, strength of strong force depends on the energy of the interaction or the distance between particles. At lower energies or longer distances: a) color charge strength increases; b) strong force becomes 'stronger'; c) nucleons can be considered as fundamental nuclear particles and quarks seem to be strongly bound within the nucleons leading to 'Quark confinement'. At high energies or short distances: a) color charge strength decreases; $b$ ) strong force gets 'weaker';3) colliding protons generate 'scattered free quarks' leading to 'Quark Asymptotic freedom'. Based on these points, low energy nuclear scientists assume 'strong interaction' as a strange nuclear interaction associated with binding of nucleons and its implications were not considered. High energy nuclear scientists consider nucleons as composite states of quarks and try to understand the nature and strength of strong interaction $\left(\alpha_{s}\right)$ at sub nuclear level. According to QCD, $\left(\alpha_{s}\right)$ decreases with increasing interaction energy. By definition, at low energy scales, $\alpha_{s} \cong 1$ and by experiments and observations, at 80 to $90 \mathrm{GeV}$ energy scales, $\alpha_{s} \cong 0.1186$.

At this juncture, one important question to be answered and reviewed at basic level is: How to understand nuclear interactions in terms of sub nuclear interactions? Unfortunately, 1) At $1.2 \mathrm{fm}$ scale, there is no practical evidence or applications for the basic definition of $\alpha_{s} \cong 1$.2) With current concepts of QCD, one cannot explain the observed nuclear binding energy scheme. 3) Famous nuclear models like, Liquid drop model and Fermi gas model [3-6] are lagging in answering this question. To find a way, we would like to suggest that, by implementing the 'strong coupling constant' of magnitude 0.1186 - in low energy nuclear physics, nuclear charge radius, Fermi's weak coupling constant and strong coupling constant can be studied in a unified picture. Proceeding further, close to beta stability line, nuclear binding energy can be addressed with a single energy coefficient of magnitude (8.9 to 10.0) $\mathrm{MeV}[7-10]$. 


\section{Three heuristic relations}

In this section we present three heuristic relations. We believe that, they are having deep inner meaning at very fundamental level and they can be derived with further research.

\section{Relation-1:}

$$
R_{0} \cong\left(\frac{m_{p}}{m_{e}}\right) \sqrt{\frac{F_{W}}{\hbar c}} \cong 1.23742 \mathrm{fm}
$$

Here, $F_{W}=$ Fermi's weak coupling constant $=1.43586 \times 10^{-62} \mathrm{~J}_{\mathrm{m}}{ }^{3} ; m_{e}=$ Rest mass of electron; $m_{p}=$ Rest mass of proton; $R_{0}=$ Nuclear charge radius.

\section{Relation-2:}

$$
R_{0} \cong\left(\frac{1}{\sqrt{\alpha_{s}}}\right)\left[\frac{\hbar}{m_{p} c}+\frac{\hbar}{m_{p} c}\right] \cong\left(\frac{1}{\sqrt{\alpha_{s}}}\right)\left[\frac{2 \hbar}{m_{p} c}\right] \cong 1.2214 \mathrm{fm}
$$

$m_{p}=$ Rest mass of proton; $m_{n}=$ Rest mass of neutron; $\alpha_{s}=$ Strong coupling constant.

Relation-3: From relations (1) and (2),

$$
\alpha_{s} F_{W} \cong \frac{4 \hbar^{3} m_{e}^{2}}{m_{p}^{4} c}
$$

Based on relation (3),

$$
\begin{gathered}
F_{W} \cong \frac{4 \hbar^{3} m_{e}^{2}}{\alpha_{s} m_{p}^{4} c} \cong 1.399 \times 10^{-62}{\mathrm{~J} . \mathrm{m}^{3}}^{2} \\
\alpha_{s} \cong \frac{4 \hbar^{3} m_{e}^{2}}{m_{p}^{4} c F_{W}} \cong\left(\frac{m_{e}}{m_{p}}\right)^{2}\left(\frac{4 \hbar^{3}}{m_{p}^{2} c F_{W}}\right) \cong 0.115543
\end{gathered}
$$

Based on this relation and considering relativistic energy of proton, it is possible to show that, $\alpha_{s} \propto\left\{\left[1-(v / c)^{2}\right] / m_{p}^{2}\right\}$. Qualitatively, this kind of observation seems to be in-line with modern QCD concepts.

\section{Discussion}

In our previous publications $[7,8]$, we proposed that, close to the beta stability line, nuclear binding energy can be addressed with a single energy coefficient, $B_{0}$ of the order of $10.0 \mathrm{MeV}$. With the proposed relations, it can be expressed as:

$$
\begin{aligned}
B_{0} & \cong\left(\frac{1}{\alpha_{s}}\right)\left[\frac{e^{2}}{4 \pi \varepsilon_{0} R_{0}}\right] \cong\left(\frac{1}{\alpha_{s}}\right)\left(\frac{m_{e}}{m_{p}}\right)\left(\frac{e^{2}}{4 \pi \varepsilon_{0}}\right) \sqrt{\frac{\hbar c}{F_{W}}} \\
& \cong \frac{e^{2} m_{p}^{3} c^{2}}{16 \pi \varepsilon_{0} \hbar^{2} m_{e}} \sqrt{\frac{F_{W}}{\hbar c}} \cong 10.0715 \mathrm{MeV}
\end{aligned}
$$

With this energy unit, close to beta stability line, stable mass number of $\mathrm{Z}$ can be addressed with, 


$$
\left.\begin{array}{l}
A_{s} \cong(2 Z)+(\beta Z)^{2} \\
N_{s} \cong Z+(\beta Z)^{2}
\end{array}\right\}
$$

where,

$$
\left.\begin{array}{l}
\beta \cong\left(\frac{3}{5}\right)\left[\frac{e^{2}}{4 \pi \varepsilon_{0} R_{0}}\right] \div\left\{\left(\frac{1}{\alpha_{s}}\right)\left[\frac{e^{2}}{4 \pi \varepsilon_{0} R_{0}}\right]-\left[\frac{e^{2}}{4 \pi \varepsilon_{0} R_{0}}\right]\right\} \\
\cong \frac{0.71 \mathrm{MeV}}{(10.07-1.188) \mathrm{MeV}} \cong \frac{0.71 \mathrm{MeV}}{8.89 \mathrm{MeV}}\left[\frac{3}{5}\left(\frac{\alpha_{s}}{1-\alpha_{s}}\right)\right] \cong 0.08
\end{array}\right\}
$$

Based on the new integrated model proposed by N. Ghahramany et al $[9,10]$ and with reference to relation (7), it is possible to show that, $Z \cong(40$ to 83$)$, close to the beta stability line,

$$
(B)_{A_{s}} \cong\left[A_{s}-\left(\frac{N_{s}^{2}-Z^{2}}{3 Z}\right)\right] \times 9.5 \mathrm{MeV} \cong\left[A_{s}-\left(\frac{\beta^{2} Z A_{s}}{3}\right)\right] \times 9.5 \mathrm{MeV}
$$

where, $\left[\frac{N_{s}^{2}-Z^{2}}{Z}\right] \cong \beta^{2} Z A_{s}$. Based on this strange and simple relation and with reference to our recent publications $[7,8]$ and first four terms of the semi empirical mass formula (SEMF), close to the beta stability line, for $(Z=2$ to 100$)$, it is possible to show that,

$$
\begin{aligned}
& (B)_{A_{s}} \cong\left[A_{s}-A_{s}^{1 / 3}-\frac{\beta^{2} A_{s} \sqrt{N_{s} Z}}{3.42}-1\right] \times 10.07 \mathrm{MeV} \\
& \text { where, }\left(\frac{\beta^{2}}{3.42}\right) \approx \alpha_{s}\left(\frac{a_{c}}{2 a_{a}}\right) .
\end{aligned}
$$

It is for further study. According to PDG data [11], up quark mass is $2.15 \mathrm{MeV} / c^{2}$ and down quark mass is 4.7 $\mathrm{MeV} / c^{2}$. With reference to proton and neutron, for these two basic quark masses,

$$
\left.\begin{array}{l}
\frac{\left(2 m_{u}+m_{d}\right)+\left(m_{u}+2 m_{d}\right)}{2} \cong 10.275 \mathrm{MeV}, \\
\sqrt{\left(2 m_{u}+m_{d}\right)\left(m_{u}+2 m_{d}\right)} \cong 10.2 \mathrm{MeV} \\
\text { and } \frac{2\left(2 m_{u}+m_{d}\right)\left(m_{u}+2 m_{d}\right)}{\left[\left(2 m_{u}+m_{d}\right)+\left(m_{u}+2 m_{d}\right)\right]} \cong 10.12 \mathrm{MeV}
\end{array}\right\}
$$

Based on relations (10) and (11), it is also possible to interpret that, nuclear binding energy is proportional to mean mass of $\left[\left(2 m_{u}+m_{d}\right),\left(m_{u}+2 m_{d}\right)\right]$. This idea seems to be in line with the new model proposed by N. Ghahramany et al in 2011 [12].

With reference to SEMF, close to the beta stability line, it is also possible to show that,

$$
\frac{\left(A_{s}-2 Z\right)^{2}}{A_{s}} \cong\left(\beta^{4} A_{s} N_{s} \sqrt{Z}\right)
$$


Let, $\left\{\begin{array}{l}a_{v} \cong a_{s} \cong a_{a} \approx 14.8 \mathrm{MeV} \approx(3 / 2) \times 10.0 \mathrm{MeV} \\ \text { and } a_{c} \cong 0.71 \mathrm{MeV}\end{array}\right\}$.

If so,

$$
(B)_{A_{s}} \approx\left[A_{s}-A_{s}^{2 / 3}-0.0473\left[\frac{Z(Z-1)}{A_{s}^{1 / 3}}\right]-\left(\beta^{4} A_{s} N_{s} \sqrt{Z}\right)\right] \times 14.8 \mathrm{MeV}
$$

In comparison with SEMF, by replacing $A_{S}$ with $A$ in relation (13) and by considering a multiplication factor of the kind $\left(\frac{A_{s}}{A}\right)^{1-(Z / A)}$ associated with each term, binding energy of $A$ can be estimated approximately. For $Z=50$ and $A=100$ to 136 , estimated binding energy range is (829 to 1120$) \mathrm{MeV}$ and can be compared with reference binding energy[4] range of (806 to 1105$) \mathrm{MeV}$.

For relations (10) and (13), see figure 1 (red and violet curves respectively) for the estimated binding energy per nucleon close to beta stability line of $\quad Z=2$ to 100 compared with first four terms of the semi empirical mass formula (Green curve) where $a_{v} \cong 15.77 \mathrm{MeV}, a_{s} \cong 18.34 \mathrm{MeV}, a_{a} \cong 23.2 \mathrm{MeV}$ and $a_{c} \cong 0.71 \mathrm{MeV}$.

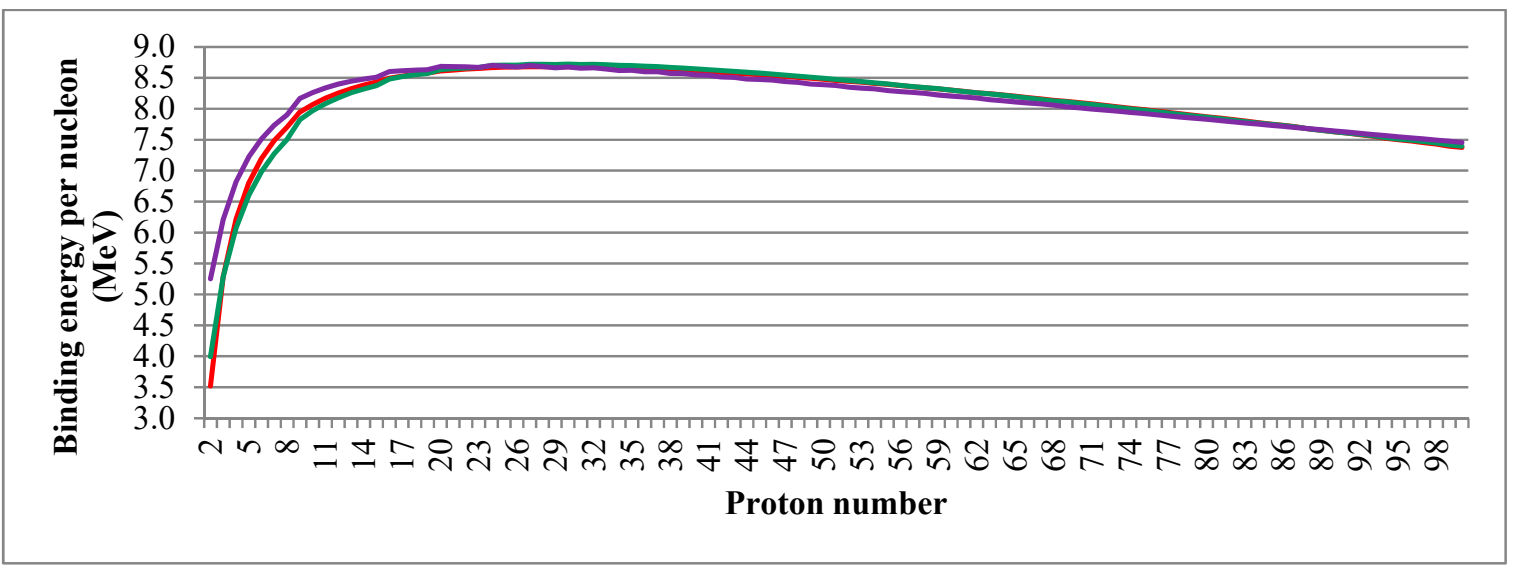

Figure 1: Binding energy per nucleon close to beta stability line of $Z=2$ to 100

\section{Conclusions}

Based on the proposed relations (1) to (12), beta decay and existence of massive electroweak bosons, we would like to suggest that, unidentified physics is happening at nuclear scale and needs a serious study at basic level.

\section{Acknowledgements}

Author Seshavatharam is indebted to professors shri M. Nagaphani Sarma, Chairman, shri K.V. Krishna Murthy, former Chairman, Institute of Scientific Research in Vedas (I-SERVE), Hyderabad, India and Shri K.V.R.S. Murthy, former scientist IICT (CSIR), Govt. of India, Director, Research and Development, I-SERVE, for their valuable guidance and great support in developing this subject.

\section{References}

[1] S. Bethke and G.P. Salam. Quantum chromodynamics. K.A. Olive et al. (Particle Data Group), Chin. Phys. C, 38, 090001 (2014) and 2015 update.

[2] David J. Gross. Twenty Five Years of Asymptotic Freedom. Nucl.Phys.Proc.Suppl. 74 (1999) 426-446 
[3] Weizsäcker, Carl Friedrich von, On the theory of nuclear masses; Journal of Physics 96 (1935) pages 431 - 458.

[4] W. D. Myers et al. Table of Nuclear Masses according to the 1994 Thomas-Fermi Model.(from nsdssd.lbl.gov)

[5] P. Roy Chowdhury et al. Modified Bethe-Weizsacker mass formula with isotonic shift and new driplines. Modern Physics Letters A 20.21 1605-1618 (2005)

[6] J.A. Maruhn et al., Simple Models of Many-Fermion Systems, Springer-Verlag Berlin Heidelberg 2010. Chapter 2, page:45-70.

[7] U. V. S Seshavatharam, S. Lakshminarayana. Simplified Form of the Semi-empirical Mass Formula. Prespacetime Journal. (2017). 8(7): 881-810.

[8] U. V. S Seshavatharam, S. Lakshminarayana. On the role of strong coupling constant and nucleons in understanding nuclear stability and binding energy. Journal of Nuclear Sciences. (2017); 4(1): 7-18.

[9] Ghahramany et al. New approach to nuclear binding energy in integrated nuclear model. Journal of Theoretical and Applied Physics 2012, 6:3.

[10]N. Ghahramany et al. Stability and Mass Parabola in Integrated Nuclear Model. Universal Journal of Physics and Application. (2013); 1(1): 18-25.

[11] A.V. Manohar, C.T. Sachrajda. Quark masses. C. Patrignani et al. (Particle Data Group), Chin. Phys. 2016; C( 40), 100001.

[12]N. Ghahramanyet al. New scheme of nuclide and nuclear binding energy from quark-like model. Iranian Journal of Science \& Technology. 2011; A3: 201-208. 